\title{
Cost-effectiveness analysis comparing intensity-modulated radiotherapy with conformational radiotherapy (3D-RT) for prostate cancer in the brazilian health system

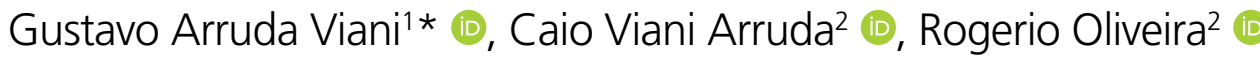

\begin{abstract}
SUMMARY
OBJECTIVE: The intensity-modulated radiotherapy (IMRT) has been established as the standard external-beam radiation technique to treat prostate cancer in several countries. In Brazil, the public health system and the National Health Agency do not reimburse its utilization. This study compared the cost-effectiveness of IMRT and tridimensional radiotherapy (3D-RT) from a payer's perspective.

METHODS: We built a Markov model to delineate the health states after treatment with IMRT and 3D-RT. The treatment-related toxicity data were extracted from the literature. The sensitivity analyses were performed over potential parameters.

RESULTS: The incremental cost of IMRT was R\$5,553.78. At a time horizon of 5 years, the quality-adjusted life expectancy after IMRT was higher than 3D-RT. The incremental cost-effectiveness ratio (ICER) of IMRT over 3D-RT was R\$-296,74/quality-adjusted life year (QALY). Therefore, IMRT was the dominant strategy, which depended on the value of interventional procedures for severe toxicity and the difference between IMRT and 3D-RT reimbursement. The IMRT was still most likely to be cost-effective at a willingness-to-pay R\$100,000/ QALY, with a net monetary benefit (NMB) superior to 3D-RT after $R \$ 50,000.00$, resulting in a difference of $R \$ 220.000,00$ after 5 years. CONCLUSIONS: IMRT generated more values than 3D-RT for external-beam treatment. Given its potential to reduce late toxicity with hypofractionation, these data reinforce its incorporation to treat prostate cancer in the Brazilian health system from a payer's perspective. KEYWORDS: Prostate Cancer. Cost-effectiveness. Intensity-modulated radiotherapy.
\end{abstract}

\section{INTRODUCTION}

Radiotherapy plays a crucial role in the management of prostate cancer $^{1}$. In the last decades, radiotherapy has passed through a significant technological advance with the development of intensity-modulated radiotherapy $(\text { IMRT })^{2-4}$. IMRT has the capacity of delivering the prescription dose to the target volume while sparing the normal tissues around the gland ${ }^{2}$.

The retrospective cohorts and randomized clinical trials showed that IMRT produces similar biochemical control with reduced late toxicity and better quality of life ${ }^{5-9}$. In the RTOG 0126, patients who received IMRT with conventional fractionation had a reduction in any late toxicity compared with tridimensional radiotherapy (3D-RT) ${ }^{9}$. The only randomized trial comparing IMRT with 3D-RT using moderated hypofractionation for prostate cancer showed a significant benefit for late gastrointestinal (GI) toxicity grade 2 or higher and better quality of life ${ }^{8}$.

Based on these outcomes, IMRT has been recommended as the standard technique for the patients with prostate cancer ${ }^{10,11}$.

\footnotetext{
'Faculdade de Medicina de Ribeirão Preto da Universidade de São Paulo - Ribeirão Preto (SP), Brazil.

${ }^{2}$ Instituto de Biociência da Universidade do Estado de São Paulo - Botucatu (SP), Brazil.

*Corresponding author: gusviani@gmail.com

Conflicts of interest: the authors declare there is no conflicts of interest. Funding: none.

Received on February 04, 2021. Accepted on March 142021.
} 
The American Society of Radiation Oncology (ASTRO) and National Comprehensive Cancer Network (NCCN) also suggest that the IMRT is considered to deliver radiation to the prostate $^{10,11}$. However, in Brazil, the public health system (Sistema Unico de Saúde [SUS]) and health insurance do not reimburse the IMRT. The Brazilian Supplemental Health System (Agência Nacional de Saúde Supplementary [ANS]) does not have the IMRT registered ${ }^{3}$. Consequently, it is not reimbursed by health insurance companies.

The IMRT is more expensive and time-consuming, requiring much attention from the radiation oncologist and medical physicist in all steps involved in the process for the patient be and adequately treated at the machine ${ }^{3}$. However, even being more expensive, health systems from other countries, such as Canada and England, have incorporated the technique into their clinical practice due to its better cost-effectiveness ${ }^{12}$.

In the Brazilian context, to the best of our knowledge, no study was published evaluating the cost-effectiveness of IMRT over 3D-RT. Therefore, this study aims to perform a cost-effective analysis with Markov chain comparing IMRT versus 3D-RT with a simulation using the data available in the medical literature on the perspective of health care system.

\section{METHODS}

A Markov model was designed to simulate the clinical outcomes of a 62-year-old man with localized prostate cancer. The Markov model allows us to hypothetically simulate the cohorts of patients with transitions between different health states using fixed increments of time ${ }^{13}$. In other words, this model considers a hypothetical patient with localized prostate cancer and simulates the possibility of the occurrence of events after treatment. Over the course, the patient may develop treatment-related toxicity, disease recurrence, or death from any causes; each of these events is correlated with a cost and a utility. The patient is followed up until death, and this process is repeated thousands of times to estimate an average cost and utility for each treatment. The two radiation techniques were compared using the Markov model, namely, IMRT to 70 Gy in 2.8 Gy per fraction and 3D-RT with the same total dose or dose per fraction. We assumed that patients started in a well state, i.e., with no evidence of disease or symptom like or similar to the radiation collateral effects. The analysis was conducted from the payer's perspective. The cycle length was calculated per month, and we used a lifetime time horizon of 5 years.

\section{Toxicity and assumptions}

All probabilities were extracted from the randomized clinical trials identified in the systematic search on the electronic databases ${ }^{8,9}$. Based on the outcomes of randomized clinical trials, we have assumed that biochemical failure and death was similar between IMRT and 3D-RT. Toxicities were defined as the probability of developing grade 2 or higher GI/genitourinary (GU) toxicity by the toxicity criteria of the Radiation Therapist Oncology Group (RTOG) or Common Toxicity Criteria (CTC). Patients could have any combination of these toxicities, assuming that the development of any toxicity was independent of the others. All toxicities from treatment were assumed to develop within 5 years from the treatment. Table 1 describes the rates of toxicities extracted from the randomized clinical trials.

\section{Costs}

The baseline costs of IMRT and 3D-CRT were extracted from the CBHPM payment schedule, as described in Table 1. Costs for the management of GI and GU toxicities were also extracted from the CBHPM 2016 schedule, as shown in Table 1. Routine follow-up visits, including periodic PSA control, were not included in the model once IMRT and 3D-RT were assumed to be identical ${ }^{14}$.

\section{Utility}

The quality-adjusted life year (QALY) is considered the standard for the cost-effective analysis studies. It was used as the effectiveness measure. The QALY is defined as a combination of quality of life and length of life. In the QALY, each year of life is weighted by a factor that effectively reflects the quality of life ${ }^{13}$. This factor is determined by a health state utility. A utility is an econometric measure that a patient gives a value to his/her health status ranging from 0 (death) to 1 (perfect health). In this model, each health state, such as GI, is associated with a utility. A QALY is estimated by multiplying the length of life in such a health state by its utility. We used the utilities for health states reported by Sweart et al. ${ }^{15}$, as detailed in Table 1 .

\section{Discounting and analysis}

An annual discount of 5\% on the costs and QALYs was given. All the analyses were performed by using TreeAge Pro software. The effect of adjusting the assumptions in the model was evaluated by using the sensitivity analysis. The difference between the cost of IMRT and 3D-RT, \% grade 2 or higher GI toxicity, \% grade 2 or higher GU toxicity, and price and number of medical procedures were ranged in the one-way sensitivity analysis. When a radiation modality was both more effective (higher QALYs) and less costly, it was considered dominating the other strategies. If a radiotherapy modality was not only more effective but also more costly, 
the incremental cost-effectiveness ratio (ICER) was described. We also performed a net monetary benefit (NMB) to represent the value of an intervention in monetary terms in the case of the health system was the willingness to pay a threshold for a unit of QALY.

\section{RESULTS}

The Markov model was calibrated to replicate the rates of grade 2 or higher GI and GU toxicities in patients treated with IMRT and 3D-CRT, producing similar probabilities of complications to the extracted data from randomized trials, which was similar to

Table 1. Description of the parameters, values, and amounts considered in the Markov chain model including the costs, toxicity, and health transitions extracted from the literature for intensity-modulated radiotherapy and tridimensional radiotherapy.

\begin{tabular}{|c|c|c|c|}
\hline Radiotherapy cost & Value & Unity & Reference \\
\hline Medical visits & $\mathrm{R} \$ 77.00$ & 1 & CBHPM, $2016^{14}$ \\
\hline Planning and simulation CT & $\mathrm{R} \$ 339.14$ & 1 & CBHPM, $2016^{14}$ \\
\hline RT device to treat & $\mathrm{R} \$ 564.64$ & 1 & CBHPM, $2016^{14}$ \\
\hline Computerized complex planning & $\mathrm{R} \$ 1,457.48$ & 1 & CBHPM, $2016^{14}$ \\
\hline Total cost IMRT & $\mathrm{R} \$ 22,440.21$ & & CBHPM, $2016^{14}$ \\
\hline Total cost 3D-RT & $R \$ 16,906.48$ & 1 & CBHPM, $2016^{14}$ \\
\hline $\begin{array}{l}\text { Fractionation } \\
\text { Conventional hypofractionated }\end{array}$ & $\begin{array}{l}\text { Number of fractions } \\
39 \\
25 \\
\end{array}$ & $\begin{array}{c}\text { Number of treatment } \\
1\end{array}$ & CBHPM, $2016^{14}$ \\
\hline Treatment complication costs & Frequency/year & Monetary value & Reference \\
\hline Medical visits posttreatment & 3 & $\mathrm{R} \$ 77.00$ & CBHPM, 2016 \\
\hline $\begin{array}{l}\text { Medical treatment for grade } 2 \text { or higher Gl } \\
\text { Follow-up visits } \\
\text { Medical drugs } \\
\text { (sulfasalazine) }\end{array}$ & $\begin{array}{c}3 \\
12\end{array}$ & $\begin{array}{l}R \$ 77.00 \\
R \$ 90.00\end{array}$ & CBHPM, $2016^{14}$ \\
\hline $\begin{array}{l}\text { Interventional treatment for grade } 2 \text { or } \\
\text { higher } \mathrm{Gl} \\
\text { Interventional procedure (argon laser) }\end{array}$ & 2 & $\mathrm{R} \$ 917.00$ & CBHPM, $2016^{14}$ \\
\hline Health states & Value & Standard deviation & Reference \\
\hline Radiotherapy & 0.73 & 0.2 & Stewart et al. $2005^{15}$ \\
\hline Death & 0 & - & By definition \\
\hline GI radiation side effects & 0.71 & 0.09 & Stewart et al. $2005^{15}$ \\
\hline GU radiation side effects & 0.79 & 0.23 & Stewart et al. $2005^{15}$ \\
\hline Both & 0.70 & 0.24 & Stewart et al. $2005^{15}$ \\
\hline Acute toxicity & $\begin{array}{l}\text { Grade } 2 \text { or higher Gl } \\
\text { acute toxicity }\end{array}$ & $\begin{array}{l}\text { Grade } 2 \text { or higher } \\
\text { GU acute toxicity }\end{array}$ & Reference \\
\hline Hypofractionation & $\begin{array}{l}24 \% \text { 3D-RT } \\
7 \% \text { IMRT }\end{array}$ & $\begin{array}{l}27 \% \text { 3D-RT } \\
9 \% \text { IMRT }\end{array}$ & Viani et al. $2016^{8}$ \\
\hline Conventional fractionation & $\begin{array}{l}18 \% \text { 3D-RT } \\
3.8 \% \text { IMRT }\end{array}$ & $\begin{array}{l}13 \% \text { 3D-RT } \\
8.5 \% \text { IMRT }\end{array}$ & Michalski et al. $2013^{9}$ \\
\hline Late toxicity & $\begin{array}{c}\text { Grade } 2 \text { or higher } \mathrm{Gl} \\
\text { acute toxicity }\end{array}$ & $\begin{array}{l}\text { Grade } 2 \text { or higher } \\
\text { GU acute toxicity }\end{array}$ & Reference \\
\hline Hypofractionation & $\begin{array}{l}64 \% \text { 3D-RT } \\
21.7 \% \text { IMRT }\end{array}$ & $\begin{array}{l}37 \% \text { 3D-RT } \\
12.3 \% \text { IMRT }\end{array}$ & Viani et al. $2016^{8}$ \\
\hline Conventional fractionation & $\begin{array}{l}20.8 \% \text { 3D-RT } \\
14.8 \% \text { IMRT }\end{array}$ & $\begin{array}{l}13.4 \% \text { 3D-RT } \\
7.4 \% \text { IMRT }\end{array}$ & Michalski et al. $2013^{9}$ \\
\hline
\end{tabular}

CT: computadorized tomography; RT: radiotherapy; IMRT: intensity-modulated radiotherapy; 3D-RT: tridimensional radiotherapy; GI: gastrointestinal; GU: genitourinary. 
their targeted probabilities. Initially, the incremental cost of IMRT was R \$ 5,533.73, i.e., 32\% higher than 3D-RT. The cost-effectiveness analysis, considering the 5-year horizon, showed that the IMRT resulted in R \$ 5,959.00, and the 3D-RT generated a value of $R \$ 1,2437.00$, as demonstrated in Figure 1A.

The monetary value with IMRT was initially higher than the 3D-RT; however, after the period analysis, the 3D-RT generated a much higher expense than the IMRT, reaching its value and exceeding it, leaving a difference of $\mathrm{R} \$ 6,478.00$.

Regarding QALYs, IMRT presented a value of 41.08 and 3D-RT, 19.25; therefore, IMRT generated an increase in the QALYs of 21.8, and it means that IMRT provided a higher quality of life for the patient over 5 years who experienced fewer complications as a result of the radiotherapy

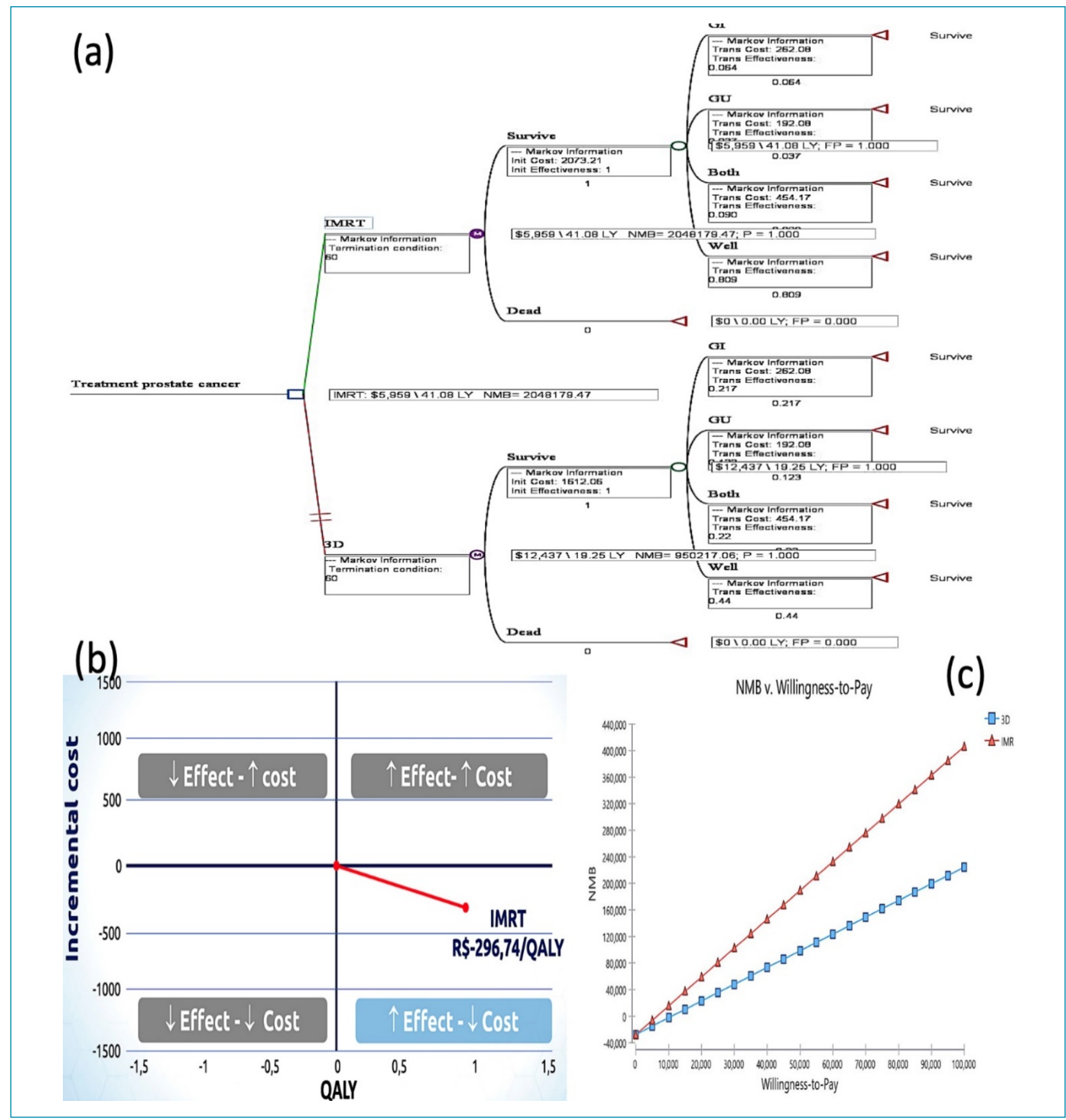

Figure 1. (a) Outcomes from the Markov chain model; (b) plan of cost-effectiveness showing intensity-modulated radiotherapy as dominant strategy; and (c) net monetary benefit comparing tridimensional radiotherapy with intensity-modulated radiotherapy. 
procedure. Combining costs and QALYs, the IMRT had a value of R\$145 per QALYs, while the 3D-RT obtained the value of $\mathrm{R} \$ 646.00$ QALYs. The ICER was $\mathrm{R} \$-296.74 /$ QALY, as shown in Figure 1B.

In the sensitivity analysis, i.e., the difference in the reimbursement between IMRT and 3D-RT, the cost of the interventional procedure to treat severe toxicity influenced the IMRT as a dominant strategy. IMRT was also highly cost-effective similar to using a conventional hypofractionated schedule, as demonstrated in Table 2. The NMB is demonstrated in Figure 1C. After a horizon of 5 years, from the perspective of the health care payer, the IMRT generated R $\$ 220,000.00$ more value than $3 \mathrm{D}-\mathrm{RT}$, mainly related to the reduction in severe late toxicities.

\section{DISCUSSION}

This study shows that IMRT is cost-effective compared with 3D-RT in the treatment of localized prostate cancer. Although IMRT has an initial incremental cost of $\mathrm{R} \$ 5,533.73$, it was associated with a lower risk of rectal and bladder toxicity, resulting in the absolute risks of severe complications very low to compensate for a significantly costlier treatment. IMRT was a strategy dominant with an ICER of R \$ -296.74/QALY. Our data agree with other cost-effective analyses from other countries that pointed in the same direction ${ }^{16-18}$.

Thus, our findings may have broad implications for the incorporation of IMRT as an option to treat localized prostate cancer in Brazil. In the sensitivity analysis, the outcomes were markedly consistent, reinforcing the argument that IMRT is cost-effective for the health payer in Brazil. The cost-effectiveness of IMRT was observed with hypo- or conventional fractionation. Besides, the cost-effectiveness had a strong relationship with the cost of rectal toxicity and with the difference between the costs of the radiation techniques. It means that from the perspective of the health payer, the IMRT at a monetary value of up to $\mathrm{R} \$ 24,000.00$ stays located at quadrant I on the plane of the cost-effective analysis, as shown in Figure 1B. Considering the values between $\mathrm{R} \$ 17,000$ and $\mathrm{R} \$ 22,000$, the IMRT was a dominant strategy. It is essential to consider that the number of medical visits during the follow-up and medical drugs was not significant in the sensitivity analysis. These points are relevant for health care payers to establish their strategy to maximize the benefits of incorporating IMRT as follows:

1. They should focus on the closer follow-up.

2. The early intervention for the GU/GI side effects may reduce the need for expensive treatment such as argon laser or surgery.

3. The integration of these actions could improve even more the cost-effectiveness of IMRT.

Considering the threshold of willingness to pay, the IMRT was cost-effective at $R \$ 50,000$ and $R \$ 100.00 /$ QALY generating a high NMB; this finding is also relevant from the payer's perspective. For example, if the health care payer is willing to invest R \$100,000.00 in the IMRT as the incorporation of technology, after a horizon of 5 years, the IMRT produced an NMB of R\$220,000.00 higher than 3D-RT, i.e., the health care payer left to spent this value with radiation complications, mainly, with toxicities.

Table 2. One-way sensitivity analysis comparing the incremental cost-effectiveness ratio of intensity-modulated radiotherapy with tridimensional radiotherapy.

\begin{tabular}{|c|c|c|c|}
\hline \multirow{2}{*}{ Parameters } & \multicolumn{3}{|c|}{ Model result } \\
\hline & Range & Lower limit & Upper limit \\
\hline \multicolumn{4}{|l|}{ Medical treatment } \\
\hline Follow-up visits & $3-12$ & IMRT* & IMRT* \\
\hline Medical drugs (sulfasalazine) & $12-24$ & IMRT* & IMRT* \\
\hline \multicolumn{4}{|l|}{ Interventional procedure } \\
\hline $\begin{array}{l}\% \text { of variation in the costs for the interventional } \\
\text { procedure for GU or Gl grade } 2 \text { toxicity }\end{array}$ & $50-100 \%$ & $\mathrm{IMRT}^{+}$ & IMRT* \\
\hline \multicolumn{4}{|l|}{ Radiotherapy techniques } \\
\hline$\%$ of radiotherapy difference between IMRT and 3D-RT & $30-50 \%$ & IMRT* & $\mathrm{IMRT}^{+}$ \\
\hline Radiation fractionation & $\begin{array}{l}\text { Conventional- } \\
\text { hypofractionated }\end{array}$ & IMRT* & IMRT* \\
\hline
\end{tabular}

GU: genitourinary; GI: gastrointestinal; IMRT: intensity-modulated radiotherapy; 3D-RT: radiotherapy; *dominant strategy with incremental costeffectiveness ratio<0/QALY; ${ }^{\dagger}$ incremental cost-effectiveness ratio<R\$1000,00/QALY. 
From the perspective of a public system, the IMRT value utilizing the CBHPM is very high compared with the current value paid for treating prostate cancer in the SUS. The Brazilian public health system does not recognize or specify the price by the radiation technique employed to treat the disease. Consequently, in Brazil, several radiation services are not stimulated to use the IMRT once the SUS pays only around $\mathrm{R} \$ 5,000.00$. It is relevant to note that the IMRT is more time-consuming, which requires better and rigorous quality control, and the patients spent more time in the machine per session. Therefore, a readjustment on the SUS reimbursement would be necessary to put the IMRT available for patients from the public health system.

Moreover, our data suggest that a wide margin of readjustment could be provided on the actual SUS reimbursement ( $R$ \$ 5,000.00), maintaining its cost-effectiveness, mainly because of the high proportion of procedures to treat severe GI/GU toxicity concerning R \$ 5,000.00. The need for surgical procedures or argon laser sessions to treat rectal bleeding, for instance, can significantly affect the cost-effectiveness of IMRT in this scenario favorably.

The incorporation of IMRT in the SUS and the Brazilian Supplemental Health System procedures is vital for prostate cancer treatment in Brazil. The novel radiation modalities, such as stereotactic body radiotherapy (SBRT), utilize the IMRT as a technical requirement to deliver ultra-hypofractionation with a dose higher than 6 Gy per fraction. Recently, a large randomized trial compared the UHFT-RT with the conventional radiotherapy showed a similar biochemical control and toxicity between the treatment delivering radiotherapy in 7 versus 39 fractions for the patients with intermediate- and high-risk prostate cancer ${ }^{19}$. SBRT is very precise and extremely comfortable and convenient to the patients, and without the IMRT, the Brazilian patients will not have access to this very effective and safe radiation modality.
The nature of our model-based analysis has some limitations. Our assumptions were based entirely on the solid outcomes for 5 years; therefore, they are more immature for an extended follow-up. However, it is reasonable to infer that the cost-effectiveness of the IMRT with hypofractionation can increase over time, mainly driven by rectal toxicity.

\section{CONCLUSIONS}

This study showed that IMRT is a cost-effective radiation modality versus the $3 \mathrm{D}-\mathrm{RT}$. Our analysis strongly suggests that IMRT should be preferred to treat the patients with prostate cancer independently of fractionation in a cost-conscious environment.

The cost-effectiveness was robust throughout the multiple sensitivity analyses. The value of reimbursement of grade 2 or higher GI/GU toxicities and the difference between IMRT and 3D-RT magnified these outcomes. Given the significant amount of men diagnosed with localized prostate cancer each year, our analysis strongly argues in favor of its incorporation by the public health system and the Brazilian Supplemental Health System (ANS).

\section{AUTHORS' CONTRIBUTIONS}

GAV: Conceptualization, Data curation, Formal analysis, Methodology, Project administration, Resources, Software, Supervision, Validation, Visualization, Writing - original draft, Writing - review \& editing. CVA: Conceptualization, Data curation, Formal analysis, Methodology, Project administration, Resources, Software, Supervision, Validation, Visualization, Writing - original draft, Writing - review \& editing. RO: Conceptualization, Data curation, Formal analysis, Methodology, Project administration, Resources, Software, Supervision, Validation, Visualization, Writing - original draft, Writing review \& editing.

\section{REFERENCES}

1. Maurice MJ, Kim SP, Abouassaly R. Current status of prostate cancer diagnosis and management in the United States. JAMA Oncol. 2016;2(11):1505-7. https://doi.org/10.1001/ jamaoncol.2016.1785

2. Fischer-Valuck BW, Rao YJ, Michalski JM. Intensity-modulated radiotherapy for prostate cancer. Transl Androl Urol 2018;7(3):297-307. https://doi.org/10.21037/tau.2017.12.16

3. Novaes PERS, Mottas RT, Lundgren MSFS. Treatment of prostate cancer with intensity modulated radiation therapy (IMRT). Rev Assoc Med Bras. 2015;61(1):8-16. https://doi. org/10.1590/1806-9282.61.01.008
4. Podder TK, Fredman ET, Ellis RJ. Advances in radiotherapy for prostate cancer treatment. In: Schatten $\mathrm{H}$, editor. Molecular \& diagnostic imaging in prostate cancer: clinical applications and treatment strategies. Cham: Springer International Publishing; 2018, p. 31-47.

5. Shimizuguchi T, Nihei K, Okano T, Machitori $Y$, Ito K, Karasawa K. A comparison of clinical outcomes between three-dimensional conformal radiotherapy and intensitymodulated radiotherapy for prostate cancer. Int J Clin Oncol. 2017;22(2):373-9. https://doi.org/10.1007/s10147016-1057-y 
6. Sveistrup J, af Rosenschöld PM, Deasy JO, Oh JH, Pommer T, Petersen $\mathrm{PM}$, et al. Improvement in toxicity in high risk prostate cancer patients treated with image-guided intensity-modulated radiotherapy compared to 3D conformal radiotherapy without daily image guidance. Radiat Oncol. 2014;9:44. https://doi.org/10.1186/1748-717X-9-44

7. Viani G, Hamamura AC, Faustino AC. Intensity modulated radiotherapy (IMRT) or conformational radiotherapy (3D-CRT) with conventional fractionation for prostate cancer: is there any clinical difference? Int Braz J Urol. 2019;45(6):1105-12. https://doi.org/10.1590/S1677-5538.IBJU.2018.0842

8. Viani GA, Viana BS, Martin JEC, Rossi BT, Zuliani G, Stefano EJ. Intensity-modulated radiotherapy reduces toxicity with similar biochemical control compared with 3-dimensional conformal radiotherapy for prostate cancer: a randomized clinical trial. Cancer. 2016;122(13):2004-11. https://doi.org/10.1002/cncr.29983

9. Michalski JM, Yan $Y$, Watkins-Bruner D, Bosch WR, Winter $K$, Galvin JM, et al. Preliminary toxicity analysis of 3-dimensional conformal radiation therapy versus intensity modulated radiation therapy on the high dose arm of the radiation therapy oncology group 0126 prostate cancer trial. Int J Radiat Oncol Biol Phys. 2013;87(5):932-8. https://doi.org/10.1016/j.jirobp.2013.07.041

10. Morgan SC, Hoffman K, Loblaw DA, Buyyounouski MK, Patton C, Barocas D, et al. Hypofractionated Radiation Therapy for Localized Prostate Cancer: executive summary of an ASTRO, ASCO, and AUA Evidence-Based Guideline. Pract Radiati Oncol. 2018;8(6):354-60. https://doi.org/10.1016/j.prro.2018.08.002

11. Mohler JL, Antonarakis ES, Armstrong AJ, D'Amico AV, Davis BJ, Dorff T, et al. Prostate cancer, version 2.2019, NCCN Clinical Practice Guidelines in Oncology. J Natl Compr Canc Netw. 2019;17(5):479-505. https://doi.org/10.6004/jnccn.2019.0023

12. National Institute for Health and Care Excellence. Prostate cancer: diagnosis and management. NICE guidance [NG131]. Guidance. [cited on 2020 Dec, 12]. Available from: https:// www.nice.org.uk/guidance/ng131
13. Hoch JS, Briggs AH, Willan AR. Something old, something new, something borrowed, something blue: a framework for the marriage of health econometrics and cost-effectiveness analysis. Health Econ. 2002;11(5):415-30. https://doi.org/10.1002/ hec. 678

14. Associação Médica Brasileira. Classificação Brasileira Hierarquizada de Procedimentos Médicos. Resolução normativa CNHM n० 23/2016. Available from: https://amb.org.br/ wp-content/uploads/2016/RN-CNHM-023_2016.pdf.

15. Stewart ST, Lenert L, Bhatnagar V, Kaplan RM. Utilities for prostate cancer health states in men aged 60 and older. Med Care. 2005;43(4):347-55. https://doi.org/10.1097/01. mlr.0000156862.33341.45

16. Konski A. Cost effectiveness of prostate cancer radiotherapy. Transl Androl Urol. 2018;7(3):371-7. https://doi.org/10.21037/ tau.2017.12.38

17. Nguyen PL, Gu X, Lipsitz SR, Choueir TK, Choi WW, Lei $Y$, et al. Cost implications of the rapid adoption of newer technologies for treating prostate cancer. J Clin Oncol. 2011;29(12):1517-24. https://doi.org/10.1200/ JCO.2010.31.1217

18. Schroeck FR, Jacobs BL, Bhayani SB, Nguyen PL, Penson $\mathrm{D}, \mathrm{Hu}$ J. Cost of new technologies in prostate cancer treatment: systematic review of costs and cost effectiveness of robotic-assisted laparoscopic prostatectomy, intensitymodulated radiotherapy, and proton beam therapy. Eur Urol. 2017;72(5):712-35. https://doi.org/10.1016/j. eururo.2017.03.028

19. Widmark A, Gunnlaugsson A, Beckman L, Thellenberg-Karlsson C, Hoyer M, Lagerlung M, et al. Ultra-hypofractionated versus conventionally fractionated radiotherapy for prostate cancer: 5-year outcomes of the HYPO-RT-PC randomised, non-inferiority, phase 3 trial. Lancet. 2019;394(10196):385-95. https://doi. org/10.1016/S0140-6736(19)31131-6 